\title{
OPTIMAL CONTROL STRATEGY FOR A HIV INFECTION MODEL VIA FOURIER SERIES
}

\author{
GH. GHANBARI AND M. H. FARAHI
}

\begin{abstract}
This paper proposes an optimal control problem, with the final goal of implementing an optimal treatment protocol which could maximize the uninfected $\mathrm{CD} 4^{+} \mathrm{T}$ cells and minimize the cost of drug, utilizing a system of ordinary differential equations which describes the interaction of the immune system with the human immunodeficiency virus(HIV). The optimal pair of control and trajectories of this nonlinear system with quadratic cost functional is obtained by Fourier series approximation. The method is based upon expanding time varying functions in the nonlinear system as their Fourier series, using the operational matrices for integration and product. The problem is reduced into a set of algebraic equations.
\end{abstract}

Mathematics Subject Classification 2000: 42A16

Additional Key Words and Phrases: HIV/AIDS, Immunotherapy, Optimal control, Fourier series operational matrix, Fourier series approximation, Fourier series product operational matrix.

\section{INTRODUCTION}

Orthogonal functions and polynomials series used frequently in various problems of dynamic systems. The main characteristic of this technique is reducing these problems to solving systems of algebraic equations, thus greatly simplifying the problem solution, and making it easy to solve. Some examples in this area, are using the Walsh functions [1], the block-pulse functions [2;3], the Chebyshev polynomials [4], the Taylor series [5; 6], the Fourier series $[7 ; 8 ; 9 ; 10]$ and the Legendre polynomials $[11 ; 12]$. In this paper Fourier series approximation is used to find the optimal pair of control and trajectories for the nonlinear optimal control system of ordinary differential equations, which describes the interaction of the immune system with the human immunodeficiency virus (HIV). The method consists of reducing the identification problem into a set of algebraic equations by first expanding the time varying functions as their Fourier series with unknown coefficients. The coefficients are determined in such a way that the necessary conditions for maximizing is imposed. By this technique, the nonlinear optimal control problem is reduced to a third order mathematical nonlinear programming problem which can be solved straightforward by many subroutines. 


\section{PROPERTIES OF FOURIER SERIES}

A function defined over the interval $a$ to $b$ may be expanded into a Fourier series as follows:

$$
f(t)=a_{0}+\sum_{n=1}^{\infty}\left\{a_{n} \cos \left(\frac{2 n \pi(t-a)}{b-a}\right)+a_{n}^{*} \sin \left(\frac{2 n \pi(t-a)}{b-a}\right)\right\},
$$

where the Fourier coefficients $a_{i}$ and $a_{i}^{*}$ are given by:

$$
\begin{aligned}
& a_{0}=\frac{1}{b-a} \int_{a}^{b} f(t) \mathrm{d} t \\
& a_{n}=\frac{2}{b-a} \int_{a}^{b} f(t) \cos \left(\frac{2 n \pi(t-a)}{b-a}\right) \mathrm{d} t, \quad n=1,2,3, \ldots, \\
& a_{n}^{*}=\frac{2}{b-a} \int_{a}^{b} f(t) \sin \left(\frac{2 n \pi(t-a)}{b-a}\right) \mathrm{d} t, \quad n=1,2,3, \ldots
\end{aligned}
$$

The series in(1) has an infinite number of terms. To obtain an approximate expression for $f(t)$, we truncate the series up to the $(2 r+1)$ th term as follows:

$$
f(t)=a_{0}+\sum_{n=1}^{r}\left\{a_{n} \cos \left(\frac{2 n \pi(t-a)}{b-a}\right)+a_{n}^{*} \sin \left(\frac{2 n \pi(t-a)}{b-a}\right)\right\}=A^{T} \phi(t),
$$

where the Fourier series coefficient vector $A$ and the Fourier series vector $\phi(t)$ are defined as:

$$
\begin{aligned}
A & =\left[\begin{array}{lllllllll}
a_{0} & a_{1} & a_{2} & \ldots & a_{r} & a_{1}^{*} & a_{2}^{*} & \ldots & a_{r}^{*}
\end{array}\right]^{T}, \\
\Phi & =\left[\begin{array}{lllllll}
\phi_{0}(t) & \phi_{1}(t) & \ldots & \phi_{r}(t) & \phi_{1}^{*}(t) & \ldots & \phi_{r}^{*}(t)
\end{array}\right]^{T},
\end{aligned}
$$

with

$$
\begin{array}{ll}
\phi_{n}(t)=\cos \left(\frac{2 n \pi(t-a)}{b-a}\right), & n=0,1,2,3, \ldots, \\
\phi_{n}^{*}(t)=\sin \left(\frac{2 n \pi(t-a)}{b-a}\right), & n=1,2,3, \ldots .
\end{array}
$$

The elements of $\Phi(t)$ are orthogonal in the interval $t \in(a, b)$. By integrating both sides of (6) from $a$ to $t$, we obtain:

$$
\begin{aligned}
& \int_{a}^{t} \phi_{0}\left(t^{\prime}\right) \mathrm{d} t^{\prime}=t-a \\
& \int_{a}^{t} \phi_{n}\left(t^{\prime}\right) \mathrm{d} t^{\prime}=\frac{b-a}{2 n \pi}\left[\phi_{n}^{*}(t)-\phi_{n}^{*}(a)\right], \quad n \geq 1, \\
& \int_{a}^{t} \phi_{n}^{*}\left(t^{\prime}\right) \mathrm{d} t^{\prime}=\frac{b-a}{2 n \pi}\left[\phi_{n}(a)-\phi_{n}(t)\right], \quad n \geq 1 .
\end{aligned}
$$

By approximating $t$ by a truncated Fourier series, the forward integral of the Fourier series vector $\Phi(t)$ can be represented by:

$$
\int_{a}^{t} \Phi\left(t^{\prime}\right) \mathrm{d} t^{\prime} \cong P \Phi(t)
$$




\section{AN EXTENSION OF LOMONOSOV'S TECHNIQUES}

where $P$ is the Fourier series operational matrix of forward integration and is given by:

$$
P=(b-a)\left[\begin{array}{cccccccccccc}
\frac{1}{2} & 0 & 0 & \ldots & 0 & 0 & \frac{-1}{\pi} & \frac{-1}{2 \pi} & \ldots & \frac{-1}{(r-1) \pi} & \frac{-1}{r \pi} \\
0 & 0 & 0 & \ldots & 0 & 0 & \frac{1}{2 \pi} & 0 & \ldots & 0 & 0 \\
\vdots & \vdots & \vdots & & \vdots & \vdots & \vdots & \vdots & & \vdots & \vdots \\
0 & 0 & 0 & \ldots & 0 & 0 & 0 & 0 & \ldots & \frac{1}{2(r-1) \pi} & 0 \\
0 & 0 & 0 & \ldots & 0 & 0 & 0 & 0 & \ldots & 0 & \frac{1}{2 r \pi} \\
\frac{1}{2 \pi} & \frac{-1}{2 \pi} & 0 & \ldots & 0 & 0 & 0 & 0 & \ldots & 0 & 0 \\
\frac{1}{4 \pi} & 0 & \frac{-1}{4 \pi} & \ldots & 0 & 0 & 0 & 0 & \ldots & 0 & 0 \\
\vdots & \vdots & \vdots & & \vdots & \vdots & \vdots & \vdots & & \vdots & \vdots \\
\frac{1}{2 r \pi} & 0 & 0 & \ldots & 0 & \frac{-1}{2 r \pi} & 0 & 0 & \ldots & 0 & 0
\end{array}\right] .
$$

Also, we can obtain (See [7]):

$$
D=\int_{a}^{b} \Phi(t) \Phi^{T}(t) \mathrm{d} t=(b-a)\left[\begin{array}{cccccc}
1 & & & & & \\
& \frac{1}{2} & & & & \\
& & \frac{1}{2} & & & 0 \\
& & \frac{1}{2} & & \\
& & & & \ddots & \\
& & & & & \frac{1}{2}
\end{array}\right] .
$$

Also,

$$
W=\int_{a}^{b} \Phi(t) \mathrm{d} t=(b-a)\left[\begin{array}{c}
1 \\
0 \\
\vdots \\
0
\end{array}\right] .
$$

Using Eqs.(3) and (10) we have:

$$
\int_{a}^{t} f\left(t^{\prime}\right) \mathrm{d} t^{\prime}=\int_{a}^{t} A^{T} \Phi\left(t^{\prime}\right) \mathrm{d} t^{\prime}=A^{T} P \Phi(t) .
$$

The product $\Phi(t) \Phi^{T}(t)$ can be obtained as:

$$
\Phi \Phi^{T} A=\tilde{A} \Phi,
$$

where $\tilde{A}$ is the product operational matrix for the vector $A$ (See [8]).

\section{HIV BASIC MODEL}

The target cells of HIV infection are lymphocyte helper cells, specially CD4 ${ }^{+} \mathrm{T}$ cells. These cells become infected and begin to produce free viruses. The main fact about $\mathrm{HIV}$ infection, is reducing the count of $\mathrm{CD} 4^{+} \mathrm{T}$ cells, which have an essential role in protecting body against different pathogens. So it is important to understand the dynamics of $\mathrm{CD}^{+} \mathrm{T}$ cell count as a function of time. In HIV infection basic model, three groups of molecules are considered; Uninfected $\mathrm{CD} 4^{+} \mathrm{T}$ cells $(\mathrm{T})$, infected 
CD4 ${ }^{+} \mathrm{T}$ cells (I) and viral load (V). Biological descriptions, translation to reactions and corresponding ODE's are presented in Table1 (See[13]).

Table I. HIV basic model interactions

\begin{tabular}{|c|c|c|c|}
\hline Biological description & $\begin{array}{l}\text { Translation to reacti- } \\
\text { ons }\end{array}$ & $\begin{array}{l}\text { Reaction } \\
\text { rate }\end{array}$ & $\begin{array}{l}\text { Translation to } \\
\text { ODE }\end{array}$ \\
\hline $\mathrm{CD}^{+}{ }^{+} \mathrm{T}$ cells production & $0 \rightarrow T$ & $s$ & $\dot{T}=s$ \\
\hline $\mathrm{CD} 4^{+} \mathrm{T}$ cells natural death & $T \rightarrow 0$ & $d$ & $\dot{T}=-d T$ \\
\hline $\begin{array}{l}\mathrm{CD} 4^{+} \mathrm{T} \text { cells become infected by vi- } \\
\text { rus }\end{array}$ & $T+V \rightarrow I+V$ & $\beta$ & $\begin{array}{l}\dot{T}=-\beta T V \\
\dot{I}=\beta T V\end{array}$ \\
\hline Infected CD4 ${ }^{+} \mathrm{T}$ cells death & $I \rightarrow 0$ & $\mu$ & $\dot{I}=-\mu I$ \\
\hline $\begin{array}{l}\text { Virus replication in infected } \mathrm{CD}^{+}{ }^{+} \mathrm{T} \\
\text { cells }\end{array}$ & $I \rightarrow I+V$ & $k$ & $\dot{V}=k I$ \\
\hline Virus natural death & $V \rightarrow 0$ & $c$ & $\dot{V}=-c V$ \\
\hline
\end{tabular}

Now, according to TableI, the complete ODE model, which is sum of contributions from all reactions, is as follows:

$$
\begin{aligned}
\dot{T} & =s-d T-\beta T V, \\
\dot{I} & =\beta T V-\mu I, \\
\dot{V} & =k I-c V,
\end{aligned}
$$

where we assume (See[14]):

$$
\begin{aligned}
& s=7, d=0.007, \beta=0.00000042163, \\
& \mu=0.0999, c=0.2, k=90.67,0 \leq t \leq 1000 .
\end{aligned}
$$

By the solution of HIV propagation model (16), one can found T, I and viral load $\mathrm{V}$. The curves of the solution of basic model (16) are shown in Figure1.

\section{HIV INFECTION OPTIMAL CONTROL MODEL}

There are three convenient groups of drugs for AIDS retroviral therapy; Reverse transcriptase, Protease, and Integrase enzyme inhibitors. In this section, we study the role of reverse transcriptase inhibitors. The main action of this kind of drugs is preventing uninfected lymphocyte cells, to be infected by viral load. According to Table1, this action is equivalent to the reaction $T+V \rightarrow I+V$. So we control the first equation to prevent the transmission of uninfected cells to infected ones. This control function is called $u(t)$, where $0 \leq u(t) \leq 1$. The most drug efficiency is in the case $u \equiv 1$ which means $\mathrm{CD} 4^{+} \mathrm{T}$ cells are not infected by viral load anymore. At the other side, $u \equiv 0$ is the case which the drug does not change the disease progression. By above argument, a control system to prevent the transmission of uninfected cells to infected ones is as follows:

$$
\begin{aligned}
\dot{T} & =s-d T-\beta T V(1-u), \\
\dot{I} & =\beta T V(1-u)-\mu I, \\
\dot{V} & =k I-c V .
\end{aligned}
$$



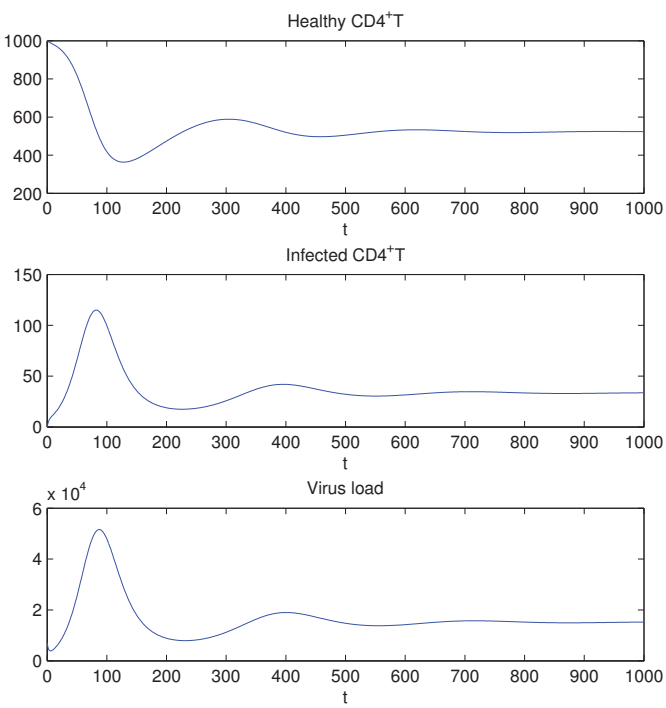

Fig. 1. The solution of basic model of HIV.

It is obvious that $T(t), I(t)$ and $V(t)$ are non-negative for all time t. For more details of three groups of control models see[15]. One can consider performance criteria for control model (17), by [16], we consider the objective functional to be defined as:

$$
J(T, u)=\int_{0}^{t_{f}}\left[T(t)-\frac{1}{2} \alpha u(t)^{2}\right] \mathrm{d} t,
$$

where is assumed $\alpha=110$ (See [16]). Our goal is maximizing the objective functional (18) subject to the control system (17); that is, maximizing the total count of $\mathrm{CD} 4^{+} \mathrm{T}$ cells and minimizing the costs of treatment by applying some RTI drugs.

\section{SOLVING HIV INFECTION OPTIMAL CONTROL MODEL, USING FOURIER SERIES}

Assume that the treatment begins when $\mathrm{CD} 4^{+} \mathrm{T}$ cells reach their minimum count, in the absence of drug. According to Figure1, T(129) = 363 is the minimum count of $\mathrm{CD}^{+} \mathrm{T}$ cells. So the treatment interval is assumed to be $[129,1000]$ day. Also, note that by Figure1, at $t=129$, we have $I(129)=57$ and $V(129)=28860$. Now, each function $T(),. I(),. V($.$) and U($.$) in the interval I=[129,1000]$, can be approximated by a Fourier series, so:

$$
T(t)=\hat{T}^{T} \Phi(t), I(t)=\hat{I}^{T} \Phi(t), V(t)=\hat{V}^{T} \Phi(t), u(t)=\hat{U}^{T} \Phi(t),
$$

where $\hat{T}, \hat{I}, \hat{V}$ and $\hat{U}$ are Fourier series coefficient vectors (all are unknown) for $T(t), I(t), V(t)$ and $u(t)$, respectively at, $t \in I$. Using Eqs.(12) and (13), one can rewrite (18) as follows: 


$$
\begin{aligned}
J(T, u) & =\int_{129}^{1000}\left[\hat{T}^{T} \Phi(t)-\frac{1}{2} \alpha \hat{U}^{T} \Phi(t) \Phi^{T}(t) \hat{U}\right] \mathrm{d} t \\
& =\hat{T}^{T} W-\frac{1}{2} \alpha \hat{U}^{T} D \hat{U},
\end{aligned}
$$

Integrating Eq.(17a) from 129 to $t$ and using Eqs.(14),(15) and (19) we have:

$$
\begin{gathered}
\int_{129}^{t} \dot{T}\left(t^{\prime}\right) \mathrm{d} t^{\prime}=T(t)-T(129) \\
=\hat{T}^{T} \Phi(t)-T_{0}^{T} \Phi(t) \\
\int_{129}^{t}[s-d T-\beta T V(1-u)] \mathrm{d} t^{\prime} \\
=\int_{129}^{t}\left[S^{T} \Phi\left(t^{\prime}\right)-d \hat{T}^{T} \Phi\left(t^{\prime}\right)-\beta \hat{T}^{T} \Phi\left(t^{\prime}\right) \Phi^{T}\left(t^{\prime}\right) \hat{V}+\beta \hat{T}^{T} \Phi\left(t^{\prime}\right) \Phi^{T}\left(t^{\prime}\right) \hat{V} \Phi^{T}\left(t^{\prime}\right) \hat{U}\right] \mathrm{d} t^{\prime} \\
=\int_{129}^{t} S^{T} \Phi\left(t^{\prime}\right) \mathrm{d} t^{\prime}-\int_{129}^{t} d \hat{T}^{T} \Phi\left(t^{\prime}\right) \mathrm{d} t^{\prime}-\int_{129}^{t} \beta \hat{T}^{T} \tilde{V} \Phi\left(t^{\prime}\right) \mathrm{d} t^{\prime}+\int_{129}^{t} \beta \hat{T}^{T} \tilde{V} \tilde{U} \Phi\left(t^{\prime}\right) \mathrm{d} t^{\prime} \\
=S^{T} P \Phi(t)-d \hat{T}^{T} P \Phi(t)-\beta \hat{T}^{T} \tilde{V} P \Phi(t)+\beta \hat{T}^{T} \tilde{V} \tilde{U} P \Phi(t),
\end{gathered}
$$

where $S$ and $T_{0}$ are Fourier series coefficient vectors of $s$ and $T(129)$, respectively; i.e. $S=[s, 0, \ldots, 0,0, \ldots, 0]^{T}, T_{0}=[T(129), 0, \ldots, 0,0, \ldots, 0]^{T}$. So, we have:

$$
\hat{T}^{T} \Phi(t)-T_{0}^{T} \Phi(t)=S^{T} P \Phi(t)-d \hat{T}^{T} P \Phi(t)-\beta \hat{T}^{T} \tilde{V} P \Phi(t)+\beta \hat{T}^{T} \tilde{V} \tilde{U} P \Phi(t) .
$$

Similarly, for Eqs.(17b) and (17c) we have:

$$
\begin{aligned}
\hat{I}^{T} \Phi(t)-I_{0}^{T} \Phi(t) & =\beta \hat{T}^{T} \tilde{V} P \Phi(t)-\beta \hat{T}^{T} \tilde{V} \tilde{U} P \Phi(t)-\mu \hat{I}^{T} P \Phi(t), \\
\hat{V}^{T} \Phi(t)-V_{0}^{T} \Phi(t) & =k \hat{I}^{T} P \Phi(t)-c \hat{V}^{T} P \Phi(t) .
\end{aligned}
$$

Eliminating $\Phi(t)$ from Eqs.(21),(22) and (23) gives:

$$
\begin{array}{r}
\hat{T}^{T}-S^{T} P+d \hat{T}^{T} P+\beta \hat{T}^{T} \tilde{V} P-\beta \hat{T}^{T} \tilde{V} \tilde{U} P-T_{0}^{T}=0 \\
\hat{I}^{T}-\beta \hat{T}^{T} \tilde{V} P+\beta \hat{T}^{T} \tilde{V} \tilde{U} P+\mu \hat{I}^{T} P-I_{0}^{T}=0 \\
\hat{V}^{T}-k \hat{I}^{T} P+c \hat{V}^{T} P-V_{0}^{T}=0 .
\end{array}
$$

To apply the constraint $0 \leq u(t) \leq 1$, one can first discretize the time interval into $(M+1)$ grid points as follows:

$$
t_{j}=129+h j, \quad j=0,1,2, \ldots, M,
$$

where $h=\frac{1000-129}{M}$. Thus $0 \leq u(t) \leq 1$, can be rewritten as:

$$
0 \leq \hat{U}^{T} \Phi\left(t_{j}\right) \leq 1, \quad j=0,1,2, \ldots, M .
$$

Similarly, $T(t), I(t)$ and $V(t) \geq 0$ can be rewritten as:

$$
\hat{T}^{T} \Phi\left(t_{j}\right) \geq 0, \hat{I}^{T} \Phi\left(t_{j}\right) \geq 0, \hat{V}^{T} \Phi\left(t_{j}\right) \geq 0, \quad j=0,1,2, \ldots, M .
$$

So, the optimal control problem now is reduced to:

$$
\operatorname{Max} \quad J(\hat{T}, \hat{U})=\hat{T}^{T} W-\frac{1}{2} \alpha \hat{U}^{T} D \hat{U}
$$



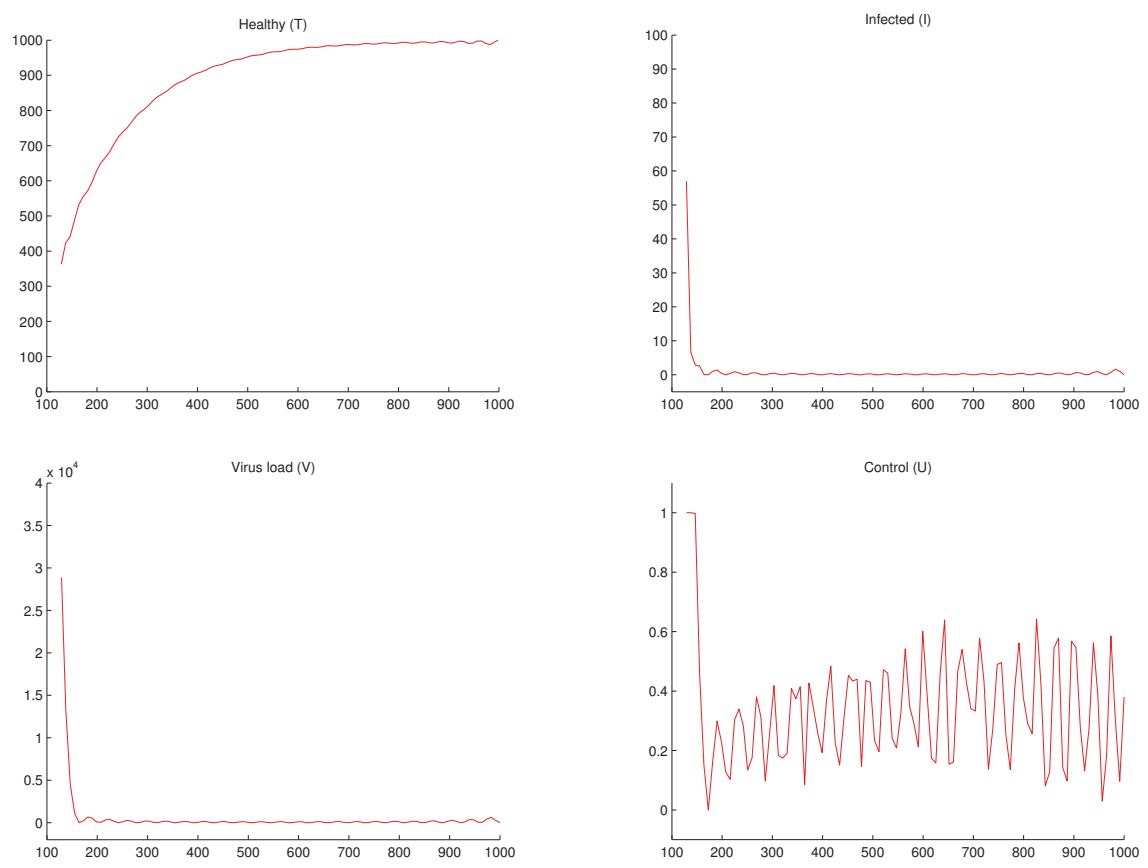

Fig. 2. The suboptimal $T(t), I(t), V(t)$ and $u(t)$.

such that:

$$
\begin{aligned}
\hat{T}^{T}-S^{T} P+d \hat{T}^{T} P+\beta \hat{T}^{T} \tilde{V} P-\beta \hat{T}^{T} \tilde{V} \tilde{U} P-T_{0}^{T} & =0 \\
\hat{I}^{T}-\beta \hat{T}^{T} \tilde{V} P+\beta \hat{T}^{T} \tilde{V} \tilde{U} P+\mu \hat{I}^{T} P-I_{0}^{T} & =0 \\
\hat{V}^{T}-k \hat{I}^{T} P+c \hat{V}^{T} P-V_{0}^{T} & =0,
\end{aligned}
$$

also:

$$
\begin{aligned}
0 \leq \hat{U}^{T} \Phi\left(t_{j}\right) \leq 1, & j=0,1,2, \ldots, M, \\
\hat{T}^{T} \Phi\left(t_{j}\right) \geq 0, \hat{I}^{T} \Phi\left(t_{j}\right) \geq 0, \hat{V}^{T} \Phi\left(t_{j}\right) \geq 0, & j=0,1,2, \ldots, M .
\end{aligned}
$$

Now, one can use Matlab software for solving above problem. The results of this optimization problem for $r=80$ and $\mathrm{M}=100$, are depicted in Figure2.

\section{CONCLUSION}

In this paper a mathematical model which shows interaction between HIV viruses and cells of human body immune system is examined. Each time varying function in the nonlinear system is expanded as its Fourier series. By using operational matrices for integration and product, the nonlinear optimal control model is reduced into a set of nonlinear algebraic equations. Since the operational matrices of integration and product contain many zero elements, its makes the method computationally attractive.

One example shows the reliability and effectiveness of the method on a HIV model. 


\section{GH. GHANBARI AND M. H. FARAHI}

\section{REFERENCES}

W. L. Chen, Y. P. Shih, Parameter estimation of bilinear systems via Walsh functions, Journal of the Franklin Institute. Vol.305, Issue 5, pp. 249-257, 1978.

K. Maleknejad, M. Shahrezaee, H.Khatamik, Numerical solution of integral equations system of the second kind by block-pulse functions, Applied mathematics and Computation, 166, pp.1524, 2005.

X. T. Wang, Yuan Min Li, Numerical solutions of integro-differential systems by hybrid of general block-pulse functions and the second Chebyshev polynomials, Applied mathematics and Computation, 209, pp. 266-272, 2009.

H. Jaddu and E. Shimemura, Solution of nonlinear optimal control problem using Chebyshev polynomials. In Proceeding of the 2nd Asian Control Conference, Soed, Korea, pages 1-417-420, 1997.

S. G. Mouroutsos, P. D. Sparis, Taylor series approach to system identification, analysis and optimal control, Journal of the Franklin Institute, Volume 319, Issue 3, pp. 359-371, 1985.

M. Gülsu, M. Sezer, A Taylor polynomial approach for solving differential-difference equations, Journal of Computational and Applied Mathematics 186, pp. 349-364, 2006.

M. Razzaghi, M. Razzaghi, Fourier series direct method for variational problems, INT. J. CONTROL, 1988, VOL. 48, NO. 3, pp. 887-895.

M. Razzaghi, Technical note, Optimal Control of Linear Time-Varying Systems via Fourier Series, JOURNAL OF OPTIMIZATION THEORY AND APPLICATIONS, 1990, Vol. 65, No. 2 .

M. L. Nagurka, V. Yen, Fourier-based optimal control of nonlinear dynamic systems, Trans. ASME J. Dyn. Syst. Meas. Control 112, pp. 17-26, 1990.

B. A. Ardekani, A. Keyhani, Identification of non-linear systems using the exponential Fourier series, Int. J. Control, VOL. 50, No. 4, pp. 1553-1558, 1989.

C. HWANG, and M. Y. CHEN, Analysis and Optimal Control of Time-Varying Linear Systems via Shifted Legendre Polynomials, International Journal of Control, Vol. 41, pp. 1317-1330, 1985 .

M. L. WANG, and R. Y. CHANG, Optimal Control of Lumped-Parameter Systems via Shifted Legendre Polynomial Approximation, Journal of Optimization Theory and Applications, Vol. 45, pp. 313-324, 1985.

M. Shirazian, M. H. Farahi, Optimal control strategy for a fully determined HIV model, ICA, 2010, pp. 15-19.

X. Xia, Estimation of HIV/AIDS parameters, Automatica, 2003, vol. 39, pp. 1983-1988.

M. Shirazian, Estimation of parameters of a mathematical model of HIV/AIDS by clinical data, MSc Dissertation, Ferdowsi university of Mashhad, 2009.

K. R. Fister and S. Lenhart, Optimizing chemotherapy in an HIV model, Journal of Differential Equations, 1998, vol. 1998, no. 32, pp. 1-12.

GH. Ghanbari, M. H. Farahi, Department of applied Mathematics, Ferdowsi University of Mashhad, Mashhad, Iran

Received October 2013 